\title{
El suelo y su relación con los servicios ecosistémicos y la seguridad alimentaria
}

The soil and its relationship with ecosystem services and food security

\section{Hernán Burbano-0rjuela ${ }^{1}$}

1 Ingeniero Agrónomo, M. Sc., Doctor Honoris Causa. Profesor titular y distinguido de la Universidad de Nariño (Jubilado); Pasto, Colombia. hernan.burbano@googlemail.com

Citar: BURBANO, H. 2016. El suelo y su relación con los servicios ecosistémicos y la seguridad alimentaria. Rev. Cienc. Agr. 33(2):117-124. doi: http://dx.doi.org/10.22267/rcia.163302.58.

\section{RESUMEN}

En este escrito, se reflexiona sobre el suelo y se destaca su importancia como recurso natural, por esta razón, se esboza su papel y los beneficios que de él se derivan para la sociedad y la naturaleza, a través de las funciones o servicios ecosistémicos que presta y de su participación para garantizar la seguridad alimentaria.

Palabras clave: antropoceno, fijación de carbono, sistema alimentario, alfabetización en suelos.

\begin{abstract}
This text discusses the importance of soil as a natural resource. It highlights the benefits derived from soil for both ecosystem and society, as a provider of ecosystem services and as a major player in guaranteeing food security.
\end{abstract}

Key Word: anthropocene, carbon fixation, food system, soils literacy.

\section{INTRODUCCIÓN}

Lo conceptuado por Hollingsworth (2015) ilustra acerca de la génesis y de los problemas inherentes a los suelos en el mundo. Este autor considera que, como los seres humanos viven en la Tierra, utilizan el suelo para obtener alimentos y para dar vigencia a la vida, porque no hay opción distinta, al menos por el momento, por ello, aclara que los suelos y la biodiversidad asociada a estos han tomado a la naturaleza un trabajo que se tasa en eones de tiempo, recursos que hoy hacen las veces de buffer frente 
al cambio climático, tanto en los ecosistemas como en los agroecosistemas. Sin embargo, éste académico señala, cómo aquello que se toma de estos recursos para suplir las demandas de la sociedad, a través de la agricultura, va en contra de la sostenibilidad del sistema.

Si se indaga por las razones que llevan a las personas a no tener un tratamiento respetuoso con la naturaleza y con recursos como el suelo, habría que expresar, que esto va a tener su origen en esa ruptura propia de la cultura occidental que considera a la naturaleza y a la cultura, por separado. Cabe decir, entonces, que en la dimensión cultural hay que introducir cambios en los patrones de comportamiento de las personas que las lleve a respetar el suelo, sus componentes y en general la vida que habita en él (Feller, 2015).

En un contexto amplio y al decir de Castellanos (2014), aunque suene paradójico, en la relación de los seres humanos con la Tierra, el mayor peligro no reside tanto en las amenazas naturales sino en la relación del "hombre moderno" con el planeta a través de sus concepciones y de su estilo de manejo.

Es por estas realidades, que en el año 2002, surge el término "Antropoceno", para referirse a la era actual de la historia de la Tierra, cuando las acciones humanas impulsan con gran fuerza, cambios ambientales, muchos de los cuales, son indeseables para un planeta superpoblado, cada vez más caliente, con tala intensa de los bosques y desafortunadamente, con degradación del suelo. No obstante, también hay esperanza, cuando se juzga que estos retos son a la vez oportunidades para reinventar y superar la situación actual (Tate y Theng, 2014).

Un informe sobre el estado de los suelos del mundo, realizado por un equipo conformado por 200 científicos del suelo de 60 países, pone de presente que si bien en algunas regiones hay buenas señales acerca de los suelos, la mayor parte de este recurso se halla al límite o en mala o muy mala situación. El documento expresa que las amenazas más fuertes para la función del suelo son la erosión, la compactación, sellado y anegamiento, la acidificación y salinización, la contaminación, el desequilibrio de nutrientes -por exceso y por deficiencia-, la pérdida del carbono orgánico del suelo que se asocia a la producción de gases de efecto invernadero y, finalmente, la pérdida de la biodiversidad. Tan grave como lo anterior, subrayan los expertos, es que el estado de los suelos va a empeorar si no se toman unas acciones concertadas entre las naciones, la sociedad civil y las organizaciones internacionales (FAO-ITPS, 2015).

Desde otra óptica, se debe relievar que, estudios como el mencionado, indican que afortunadamente hay organizaciones que se interesan y se movilizan alrededor de la preservación de los suelos del planeta, con el apoyo de realidades y argumentos científicos que coadyuvan para dar un giro a las dificultades que experimentan los suelos del mundo.

Con estas consideraciones iniciales, que buscan delinear un contexto, se procede a destacar la importancia del suelo y cómo éste recurso natural contribuye al bienestar del planeta y de la sociedad a través de las funciones o servicios ecosistémicos que presta y de su íntima relación con la seguridad alimentaria.

\section{IMPORTANCIA DEL SUELO}

El suelo es un recurso natural finito y no renovable que presta diversos servicios ecosistémicos 0 ambientales, entre ellos y a manera de ejemplo, el relacionado con su participación en los ciclos biogeoquímicos de elementos clave para la vida como carbono, nitrógeno, fósforo, etc., que continuamente y por efecto de la energía disponible, pasan de los sistemas vivos a los componentes no vivos del planeta. No obstante, lo más conocido, es que el suelo es el asiento natural para la producción 
de alimentos y materias primas de los cuales depende la sociedad mundial (CONABIO, 2016.; Silva y Correa, 2009; OBIO, 2016; Montanarella, 2015).

Puede afirmarse, además, que el suelo influye considerablemente sobre el medio en el que se ubica y repercute en las actividades sociales y económicas de los grupos humanos que se asientan en ese territorio, grupos que ejercen diferente grado de presión sobre el suelo y que generan así afectación o deterioro de éste (Gardi et al., 2014). La historia, da cuenta de cómo el suelo ha hecho parte de la vida de los seres humanos, no obstante, que es el gran olvidado cuando se alude a los recursos naturales (Burbano, 2013a y Saavedra, 2015). Por eso, hoy se juzga, que debe haber un manejo respetuoso del suelo, no solo para incrementar la producción de alimentos sino para preservar los servicios ecosistémicos y regular el clima (FA0-ITPS, 2015)

\section{FUNCIONES O SERVICIOS ECOSISTÉMICOS DEL SUELO}

Los suelos, como parte de los ecosistemas y de los agroecosistemas, prestan importantes funciones o servicios que mantienen a estos y que apoyan las actividades sociales y económicas de las personas. En opinión de varios autores (Cram et al., 2008; Silva y Correa, 2009; Burbano, 2012, Burbano, 2013b y Gardi et al., 2014), las siguientes son las tareas que cumple el suelo en éste contexto.

Producción de alimentos y biomasa. Tal vez, la más evidente de las funciones del suelo es el soporte y suministro de nutrientes para las plantas a fin de producir alimentos y biomasa en general. Caben dos observaciones. Primera, que del suelo depende en forma directa o indirecta más del 95\% de la producción mundial de alimentos. Segunda, que la degradación del suelo es un problema mayor que amenaza la producción de alimentos en el planeta.
Escenario indispensable para los ciclos biogeoquímicos. Los ciclos biogeoquímicos que ocurren en la naturaleza, son mecanismos indispensables para que haya condiciones estables en la Tierra y para que se dé la vida. Estos ciclos que involucran elementos como carbono, nitrógeno, fósforo, azufre, entre otros. son vitales y el suelo tiene una posición central e insustituible en los mismos. Sin estos ciclos no se daría el paso continuo de los elementos químicos de los sistemas vivos del planeta a otros que no lo son.

Almacenamiento o fijación de carbono. Por la importancia que tiene el carbono y dadas las circunstancias mundiales actuales del cambio climático, surge como una función aparte del suelo, aquella que se relaciona con el carbono.

El suelo es el mayor sumidero de carbono en la naturaleza. La fijación del carbono por el suelomal llamada "secuestro de carbono" o "captura de carbono"- impide que el CO2 vaya a la atmósfera, siendo que este es uno de los gases de efecto invernadero que propician el cambio climático. Con la intermediación de las plantas y la participación de los organismos del suelo, el carbono se transforma en materia orgánica que se acumula en el suelo por amplios periodos de tiempo. Este carbono almacenado en el planeta en el primer metro de suelo, se sabe, es una y media veces superior al acumulado en la vegetación.

Almacenamiento y filtración de agua. El suelo capta, infiltra y almacena el agua en el ámbito del ciclo hidrológico y permite así la recarga de los acuíferos. En estas circunstancias, el suelo influye en la calidad del agua, ya que amortigua y atrapa ciertos contaminantes e impide que lleguen a las reservas de agua. El suelo, en estas circunstancias tiende a modular indirectamente la temperatura y la humedad, y por ello puede incidir en la mejora del aire.

Soporte de las actividades humanas y fuente de materias primas. Sobre el suelo se realizan ac- 
tividades industriales, se habilitan zonas residenciales y de infraestructura turística, se construyen carreteras y otras obras civiles. También, el suelo suministra materias primas como turba, grava, arena, arcilla o rocas, utilizadas en varios procesos productivos.

Reserva de biodiversidad. El suelo es una de las reservas más importantes de biodiversidad, por el enorme número de organismos que viven en su superficie y al interior del mismo; la abundancia es tal, que se cree, supera la establecida por encima de este cuerpo natural. Vale subrayar, que los servicios ecosistémicos que presta el suelo, dependen de las complejas comunidades de organismos presentes en este medio.

Depósito del patrimonio geológico y arqueológico. Gran parte de los restos que dan cuenta de la herencia humana y de la historia ambiental reciente de la Tierra, se hallan en el suelo. La preservación del patrimonio geológico y arqueológico va a depender de los procesos de formación y degradación del suelo y va a estar muy relacionada, por ejemplo, con los efectos de la actividad biológica y la circulación del agua que ocurren él, además de las acciones humanas de intervención en el suelo.

Entorno físico y cultural para la humanidad. El suelo sirve de base a las actividades humanas y es asimismo, un elemento del paisaje y del patrimonio cultural. El suelo permite que los seres humanos tengan a su disposición alimentos, abrigo, espacio y hábitat para soñar y realizarse como personas en ascenso, y de muchos otros elementos para su existencia.

La enumeración y descripción de las funciones que cumple, pone de presente que el suelo no solo permite que las personas dispongan de alimento sino que por el espectro de servicios ambientales que presta y promueve para beneficio de las personas, este recurso adquiere la categoría de "bien social" que por lo mismo amerita su conocimiento por parte de la sociedad mundial para que lo valore, lo proteja y lo conserve, como una obligación que resulta ser en los actuales momentos un imperativo ético. Entonces, se debe conocer y respetar el suelo para hacer el mejor uso posible de este recurso, sin olvidar que existe una relación inexorable entre el sistema natural -donde está el suelo- y el sistema socio-cultural -donde están las personas- (Insuasti y Burbano, 2013 y FCM, 2013).

\section{SUELOS Y SEGURIDAD ALIMENTARIA}

Según la FAO (2011) se considera que hay seguridad alimentaria (SA) "cuando todas las personas tienen, en todo momento, acceso físico, social y económico a alimentos suficientes, inocuos y nutritivos, que satisfacen sus necesidades energéticas diarias y preferencias alimentarias para llevar una vida activa y sana". Esta conceptualización, deja en claro que referirse a SA es algo complejo y que por lo mismo demanda la integración de muchas y diversas aproximaciones.

Si la realidad del mundo de hoy, es que la sociedad consume un $95 \%$ de alimentos que en forma directa o indirecta se producen en el suelo, es posible afirmar, que la disponibilidad de alimentos, está supeditada o depende del suelo y, por ello, es que se tiende a creer que es la única función que cumple este recurso natural. Más, para efectos de garantizar la seguridad alimentaria, la producción agrícola debe provenir de un suelo sano, que es aquel que no tienen limitaciones físicas, químicas o biológicas con una productividad agrícola sostenible y con un mínimo deterioro ambiental. Cabe agregar que un suelo sano, además, aporta a la mitigación del cambio climático porque tiene gran capacidad para fijar carbono y evitar que este elemento vaya a la atmósfera (Insuasti y Burbano, 2013 y FA0 2015a).

Se estima que la tercera parte de la superficie terrestre, está dedicada a la agricultura, para una población mundial siempre creciente que podrá llegar a los 9000 millones de personas en el 2050, 
población que ejerce una presión cada vez mayor sobre los suelos que deberán producir suficientes alimentos inocuos y nutritivos para una población que hoy, según las Naciones Unidas (2014), reside mayormente en las ciudades, 54\% y que en 2015 pasó a ocupar el $66 \%$ en los centros urbanos, con el agravante de que la población urbana tiene que comprar casi todos los alimentos que requiere. Esto quiere decir, que un tercio de la población, deberá responder por el abastecimiento mundial de alimentos. De otra parte, aunque en las últimas cinco décadas los avances en la tecnología agrícola han respondido a las retos de la seguridad alimentaria, se da el caso de muchos países, que por efectos de una producción intensiva, han empobrecido sus suelos y comprometido las demandas futuras de alimentos. Si a lo enunciado se adiciona que hay problemas con el agua y existe el efecto del cambio climático, la seguridad alimentaria de hoy y del futuro va a tener muchas dificultades (Carty y Magrath, 2013; Tate y Theng, 2014; IUSS, 2015; FA0, 2015a).

Los efectos adversos sobre la calidad del medioambiente y sobre el futuro de la producción de alimentos son atribuibles, según autores como Hall (2014), a los enfoques limitados y de corto plazo que gravitan sobre el sector agrario. Frente a nuevas demandas de alimentos, la capacidad para satisfacerlas es puesta en cuestión por problemas nuevos y complejos que afectan a un sistema agrícola mundial débil, que exige un "rediseño radical" del sistema alimentario vigente para no generar un mayor daño ambiental. Los problemas por resolver son, entre otros, la escasez mundial de fósforo -por las bajas reservas terrestres de este elemento y porque las mismas están en pocos países-, los conflictos de uso de los suelos para la producción agrícola y el cambio climático. Se pide alternativas realistas para responder, con una visión orgánica sistémica, en contraposición al esquema vigente de la revolución verde.

Los efectos del cambio climático sobre los suelos son, a juicio de la FAO (2015b), una amenaza seria para la seguridad alimentaria del planeta, en razón que la fuerte modificación de temperatura y precipitación pluvial, puede impactar a la materia orgánica del suelo (MOS) y la dinámica general del mismo, igual que a la vegetación nativa y a los cultivos establecidos sobre él. Por estas y otras razones, se insiste en una gran transformación de la agricultura y del manejo de los suelos, para propiciar el incremento del carbono orgánico del suelo (COS) con la utilización de otras opciones de agricultura que respeten los ciclos biogeoquímicos, que protejan los suelos, que promuevan la asociación de especies vegetales cultivadas y la biodiversidad, con suelos menos susceptibles a la degradación y que mantengan los servicios ecosistémicos, indispensables para aumentar la producción de alimentos.

En relación con lo anterior, Carty y Magrath (2013) consideran que "un mundo caliente es un mundo hambriento" y dicen a renglón seguido, que la seguridad alimentaria está en peligro por el cambio climático, con pronósticos nada halagüeños en la lucha contra el hambre, fenómeno que si hoy ya es una realidad tenderá a exacerbarse en los próximos años. Valga saber, que pocos días de temperaturas extremas pueden inducir daños fisiológicos y pérdida de las cosechas. Hay estudios que vaticinan que en un plazo de 20 años, las áreas dedicadas en el mundo al cultivo de maíz, arroz y trigo serán afectadas en un 31, 16 y 11\%, respectivamente, por efecto de altas temperaturas que por más de cinco días al año, podrán superar los límites de resistencia de estas especies vegetales.

Para salir al paso de estas dificultades, hay un buen número de otros enfoques agrícolas que se amigan con el manejo sostenible del suelo a fin de mejorar la productividad en el campo. Entre estas alternativas se pueden mencionar: la agroecología, la agricultura de conservación, la agricultura orgánica, la agricultura de labranza cero y la agrosilvicultura basada en sistemas agroforestales SAF (FA0, 2015a). 
Por el interés que existe en Colombia, tanto en la academia como en el campo, por dos de estas opciones, a continuación se bosquejan los fundamentos de la agroecología y los sistemas agroforestales, visiones que tienen que formar parte de los programas de educación en el sector agrario en todos sus niveles.

La agroecología (AE), en su propósito de estudiar y manejar los sistemas agrícolas se fundamenta en la teoría ecológica, con la doble intención de volverlos más productivos y conservar los recursos naturales. Propugna por los sistemas e incluye diversas tecnologías, prácticas e innovaciones, lo mismo que la valoración del conocimiento localtradicional y el de la ciencia. Privilegia las interacciones de lo agropecuario, ambiental y humano y toma en cuenta lo multidimensional del sistema alimentario. Hay una versión de la AE que habla de un contra movimiento con fuertes alianzas entre la AE y la soberanía alimentaria, quizás, esta es la tendencia que ubica la $\mathrm{AE}$ dentro de un movimiento campesino con políticas transformadores que busca la soberanía alimentaria (Holt-Giménez y Altieri, 2013; FA0, 2015a).

La agroecología debe sustentarse en una educación con visión integradora y de interacción de componentes en el espacio y en el tiempo, que al comprender los beneficios de la diversidad, los incorpore para ganar resiliencia y sostenibilidad del sistema, condiciones que hacen viable la seguridad alimentaria, aunque debe advertirse que los sistemas agroecológicos deben alcanzar altos niveles de productividad frente a los sistemas de agricultura convencional, para efectos de aceptación por parte de los productores. La educación inscrita en lo agroecológico debe ser de doble vía, que propugne por una "acción basada en el conocimiento" y por un "conocimiento basado en la acción", que invite a una acción sinérgica de la teoría y de la práctica (Francis et al., 2013).

La otra alternativa mencionada, está dada por los sistemas agroforestales que toman en cuenta siste- mas tradicionales y modernos de uso del suelo, con árboles que se manejan a la par con sistemas productivos agrícolas y ganaderos. Los árboles, cultivos y ganado en simultánea mitigan el riesgo ambiental, cubren permanentemente el suelo y lo protegen de la erosión, reducen los efectos nocivos de las inundaciones y obran como depósito de agua que benefician a los cultivos establecidos dentro del sistema. Si bien esta es una alternativa idónea, la dificultad puede estribar en la concepción mental de los agricultores que, de vieja data, solo consideran los cultivos, máximo asociados con otros cultivos pero no con árboles, como que si en los ecosistemas naturales y en forma espontánea no crecieran hierbas, plantas de porte bajo, arbustos y finalmente árboles. La educación, entonces, tendrá que jugar un papel clave para que la agroforestería se abra paso (FA0, 2015a).

En la intención de alcanzar la seguridad alimentaria en el contexto del cambio climático, la FAO (2015b) impulsa un enfoque unificado que se denomina agricultura climáticamente inteligente (CSA, por sus siglas en inglés) para que los países establezcan políticas, hagan inversiones y desarrollen técnicas para el logro de las primeras. Se considera, que éstas prácticas incrementan sosteniblemente la productividad y la resiliencia al cambio climático -en apoyo de la adaptación-, a la vez que en lo posible reducen y eliminan los gases de efecto invernadero -en apoyo de la mitigación-.

Es pertinente señalar, que los suelos podrán contribuir a garantizar la seguridad alimentaria de las personas, en la medida en que se haga buen uso de aquellos y que se neutralicen o se eviten los conflictos de uso. Aunque lo dicho pareciera ser fácil de cumplir, en la realidad y dados los intereses de orden social, económico y ambiental, su consecución no es alcanzable. Los conceptos a tener en cuenta son dos. Primero, los conflictos de uso del suelo que ocurren cuando éste se utiliza para propósitos diferentes a su vocación, por eso, se deben identificar para solucionarlos y para evitar a futuro la degradación del suelo; 
el ejemplo más claro corresponde a la utilización de suelos con vocación agrícola para explotación ganadera y viceversa. Segundo, los cambios de uso del suelo, verbigracia, deforestación, urbanización, cambio de tipo de cultivos, que van a modificar localmente el comportamiento de los fenómenos meteorológicos -frecuencia de eventos extremos como tormentas, heladas, etc.-, y las variables climatológicas -temperatura, precipitación pluvial, vientos, etc.-. La interacción de los fenómenos y factores enumerados baja la productividad de los agroecosistemas implicados y se confabula contra la seguridad alimentaria (Rodríguez et al., $2010 \mathrm{y}$ FCM, 2013).

A lo largo de este acápite se ha buscado dar una serie de razones que si bien no son exhaustivas, tratan de destacar el rol que cumple el suelo en el propósito de conseguir la seguridad alimentaria que todas las personas se merecen.

\section{CONSIDERACIONES FINALES}

Infortunadamente, los suelos en el mundo, no reciben el cuidado que ameritan, porque en este como en otros casos, es difícil si no imposible, proteger y peor querer lo que no se conoce. Habrá que emprender una alfabetización en suelos para que la sociedad mundial sepa qué es el suelo y para que así, en el futuro lo valore y lo preserve (Burbano, 2004).

Los siguientes datos ilustran acerca de la situación en que se debate el suelo. Anualmente, se pierden en el mundo 75 billones de toneladas de suelo por acción de la erosión en sus diferentes manifestaciones y por efectos de la propia agricultura, pérdidas anuales que se valoran en 400 mil millones de dólares. Sin embargo, también hay motivos para el optimismo porque crece el número de personas que están hablando de "seguridad del suelo", con un significado similar a la seguridad alimentaria y a la del agua. Los suelos, se ha expresado, cobrarán cada vez más importancia en la cultura de los pueblos (Montanarella, 2015).
Puesto todo lo anterior en un panorama más amplio hay que considerar las ideas del Papa Francisco, según las cuales las dificultades que sufren los recursos naturales, entre ellos el suelo, tienen su origen en causas mayores, como los desajustes que genera la economía mundial con sus modelos de crecimiento que no respetan el medio ambiente (S.S. Fracisco, 2015).

\section{REFERENCIAS BIBLIOGRÁFICAS}

BURBANO, H. 2004. La piel de la Tierra. Cinco reflexiones para valorar el recurso suelo. Universidad de Nariño, Pasto. $176 \mathrm{p}$.

BURBANO, H. 2012. La ciencia del suelo al servicio de la sociedad y del ambiente. En: XVI Congreso Colombiano de la Ciencia del Suelo, Riohacha, La Guajira, Octubre 2-5 de 2012. Sociedad Colombiana de la Ciencia del Suelo, Bogotá. CD ROM

BURBANO, H. 2013a. La sociedad depende del todo y las partes: naturaleza y suelo. Tendencias 14:9 - 22.

BURBANO, H. 2013b. El suelo y su importancia para la sociedad, pp. 553-594. En: Burbano, H. y Silva, F., Eds. Ciencia del Suelo. Principios Básicos. Segunda Edición. Bogotá, Sociedad Colombiana de la Ciencia del Suelo, Bogotá. 594 p.

CARTY, T.; MAGRATH, J. 2013. Adversidad creciente: cambio climático, alimentos y la lucha contra el hambre. Informe Temático de Oxfam. En: https://www.oxfam.org/ sites/www.oxfam.org/files/adversidad-creciente-cambio-climatico-alimentos-hambre-informe-es.pdf; consulta: noviembre, 2015.

CASTELLANOS, M. 2014. Uso del suelo y cambio climático. 34- 37 p. En: Memorias del II Seminario de Ciencias Ambientales SUE-Caribe y VII Seminario Internacional de Gestión Ambiental. hacia un contexto de las ciencias ambientales: Iberoamérica. Monteria, Colombia.

CONABIO. COMISIÓN NACIONAL PARA EL CONOCIMIENTO Y USO DE LA BIODIVERSIDAD. 2016. Biodiversidad Mexicana. En: Ecosistemas. Procesos ecológicos. http://www.biodiversidad.gob.mx/ecosistemas/procesose.htm; consulta: enero, 2016.

CRAM, S.; COTLER, H.; MORALES, L.; SOMMER, I.; CARMONA, E. 2008. Identificación de los servicios ambientales potenciales de los suelos en el paisaje urbano del Distrito Federal. Investigaciones Geográficas, Boletín del Instituto de Geografía, UNAM. 66: 81-104. 
FCM. FEDERACIÓN COLOMBIANA DE MUNICIPIO. 2013. Estrategias de adaptación y mitigación frente a los efectos del cambio climático en Regiones de Costa y Montaña de Colombia. Fundación Konrad Adenauer KAS- FCM, Bogotá, Colombia. 93 p.

FAO - ITPS. FOOD AND AGRICULTURE ORGANIZATION OF THE UNITED NATIONS and INTERGOVERNMENTAL TECHNICAL PANEL ON SOILS. 2015. Status of de world's soil resources. ItalyTechnical Summary. Food and Agricultural Organization of the United Nations and Intergovernmental Technical Panel on Soils, Rome, Italy. 79 p.

FAO. ORGANIZACIÓN DE LAS NACIONES UNIDAS PARA LA AGRICULTURA Y LA ALIMENTACIÓN. 2011. La seguridad alimentaria: información para la toma de decisiones. Guía práctica. En: http://www.fao.org/docrep/014/al936s/ al936s00.pdf; consulta: marzo, 2016.

FAO. ORGANIZACIÓN DE LAS NACIONES UNIDAS PARA LA AGRICULTURA Y LA ALIMENTACIÓN. 2015a. Los suelos sanos son la base para la producción de alimentos saludables. La FA0 en Acción. Fao.org/soils-2015. En: https:// www.fao.org/3/a-i4405s.pdf; consulta: enero, 2016.

FAO. ORGANIZACIÓN DE LAS NACIONES UNIDAS PARA LA AGRICULTURA Y LA ALIMENTACIÓN. 2015b. Los suelos ayudan a combatir y adaptarse al cambio climático. Trabajo No. I4737. 4 p. En: http://www.fao.org/3/a-i4737s. pdf; consulta: marzo, 2016.

FELLER, C. 2015. Soil connects nature and culture. IUSS Division 4. IUSS, Vienna, Austria. Bulletin of the International Union of Soil Sciences 127:30 - 31.

FRANCIS, C.; ARVID BRELAND, T.; OSTERGAARD, E.; LIEBLEIN, G.; MORSE, S. 2013. Aprendizaje de la agroecología basado en los fenómenos: un prerrequisito para la transdisciplinariedad y la acción responsable. Agroecología. 8(2):45 - 54.

GARDI, C.; ANGELINI, M.; BARCELÓ, S.; COMERMA, J.; CRUZ GAISTARDO, C.; ENCINA, A.; JONES, A.; KRASILNIKOV, P.; MENDONÇA, M.; MONTANARELLA, L.; MUÑIZ, O.; SCHAD, P.; VARA, M.; VARGAS, R. 2014. Atlas de suelos de América Latina y el Caribe. Comisión Europea - Oficina de Publicaciones de la Unión Europea, L-2995, Luxembourg, 176 p.

HALL, S. 2014. Soils and the future of food. Challenges and oportunities for feeding nine billion people, pp. 1735. In: the soil underfoot. Inifinite possibilities for a finite resources. Churchman, G. Jock and Land, Edward R., eds. CRC Press, Boca Raton, 421 p.
HOLLINGSWORTH, I. 2015. Connecting people with soils. En: http://www.iuss.org/files/iuss-bulletin_127_72dpi. pdf; consulta: enero, 2016.

HOLT-GIMÉNEZ, E.; ALTIERI, M. A. 2013. Agroecología, soberanía alimentaria y la nueva revolución verde. Agroecología. 8(2):65 - 72.

INSUASTI, L.; BURBANO, H. 2013. El suelo: un bien social. Ministerio de Agricultura y Desarrollo Rural-Gobernación de Nariño-Universidad de Nariño. Pasto. Colombia. 30 p.

IUSS. INTERNATIONAL UNION OF SOIL SCIENCES. 2015. Soils, food security and human health. En: http://www. iuss.org/files/iuss-bulletin_127_72dpi.pdf; consulta: enero, 2016.

MONTANARELLA, L. 2015. Agricultural policy govern our soils. Nature. 528:32 - 33.

NACIONES UNIDAS. Departamento de Asuntos Económicos y Sociales. 2014. Más de la mitad de la población vive en áreas urbanas y seguirá creciendo. En: http://www.un.org/ es/development/desa/news/population/world-urbanization-prospects-2014.html; consulta: marzo, 2016.

OBIO. OBSERVATORIO NACIONAL DE BIODIVERSIDAD. 2016. Ministerio de Ambiente y Desarrollo Sostenible de la Nación. Suelos. Buenos Aires, Argentina. En: http:// obio.ambiente.gob.ar/suelos; consulta: enero, 2016.

RODRÍGUEZ, N.; PABÓN, J. D.; BERNAL, N.; MARTÍNEZ, J. 2010. Cambio climático y su relación con el uso del suelo en los Andes colombianos. Alianza Ediprint Ltda, Bogotá. Colombia. $80 \mathrm{p}$.

SAAVEDRA, J. 2015. El suelo en nuestra vida. Agricultura e Innovación. E.A.P. Zamorano. En: https://blogzamorano. wordpress.com/2015/10/16/suelos/; consulta: febrero, 2016.

SILVA, S.; CORREA, F. 2009. Análisis de la contaminación del suelo: revisión de la normativa y posibilidades de regulación económica. Semestre Económico. 23:13-34.

S.S. FRANCISCO. 2015. Carta encíclica Laudato si. "Alabado seas". Sobre el cuidado de la casa común. Paulinas. Bogotá, Colombia. 208 p.

TATE, K.; THENG, B. K. G. 2014. Climate change. An underfoot perspectiv?, pp. 3-16 In: the soil underfoot. Inifinite possibilities for a finite resources. Churchman, G. Jock and Land, Edward R., eds. CRC Press, Boca Raton, Florida. $421 \mathrm{p}$. 